\title{
Infinite derivative gravity: non-singular cosmology \& blackhole solutions
}

\author{
Anupam Mazumdar $a, b, c *$ \\ ${ }^{a}$ Van Swinderen Institute, University of Groningen, 9747 AG Groningen, The Netherlands \\ ${ }^{b}$ Kapteyn Astronomical Institute, University of Groningen, 9700 AV Groningen, The Netherlands \\ ${ }^{c}$ Lancaster University, Lancaster, LAl 4YB, United Kingdom \\ E-mail: anupam.mazumdarerug.nl
}

\begin{abstract}
Both Einstein?s theory of General Relativity and Newton?s theory of gravity possess a short distance and small time scale catastrophe. The blackhole singularity and cosmological Big Bang singularity problems highlight that current theories of gravity are incomplete description at early times and small distances. I will discuss how one can potentially resolve these fundamental problems at a classical level and quantum level. In particular, I will discuss infinite derivative theories of gravity, where gravitational interactions become weaker in the ultraviolet, and therefore resolving some of the classical singularities, such as Big Bang and Schwarzschild singularity for compact non-singular objects with mass up to $10^{25}$ grams. In this lecture, I will discuss quantum aspects of infinite derivative gravity and discuss few aspects which can make the theory asymptotically free in the UV.
\end{abstract}

Corfu Summer Institute 2016 "School and Workshops on Elementary Particle Physics and Gravity" 31 August - 23 September, 2016

Corfu, Greece

\footnotetext{
* Speaker.
} 


\section{Introduction}

Einstein's theory of General Relativity (GR) has been extremely successful in its predictions from solar system tests to galactic scales [1], and now with the direct detection of gravitational waves [2]. However, at short distances and at small time scales the theory suffers from ultraviolet (UV) catastrophe [3]. There are classical singularities such as blackhole singularity and cosmological singularity [4]. Furthermore, at a quantum level pure gravity at one loop is renormalizable, but at higher loops it is not [3]. Many attempts have been made to understand the UV aspects of gravity in the context of perturbative quantum gravity $[5,6,7]$, asymptotic safety [8], non-perturbative approaches [9], loop quantum gravity [10], and string theory [11]. All these approaches are promising, and in particular there may be some underlying connection between all these approaches. One common thread is non-local interactions, which is prevalent in many of these approaches - such as non-perturbative approaches to quantum gravity and string theory. Here, I will show that non-local interactions in gravity is inevitable, because of covariant derivative interactions. Any corrections to Einstein-Hilbert action would comprise of higher covariant derivatives for a massless graviton. But, just brining higher covariant derivative contributions in metric does not stop the agony for gravitational interactions. In fact, it deteriorates the situation, for any spin higher derivatives harbinger classical and quantum instability. At a classical level, the Hamiltonian density can be unbounded from below due to Oströgradsky theorem [12]. At a quantum level, the new dynamical degrees of freedom will be ghost [13]. The presence of ghost makes the vacuum unstable. One may hope that such a massive ghost may be superheavy compared to the scale of an effective field theory, and so at energies below the cut-off the ghost states do not meddle with the predictions of low energy theory. However, this is indeed an opportunistic view point, and it does not help addressing some of the thorny UV issues pertaining to classical singularities.

A very well-known example in this context is the quadratic curvature gravity [14], pure gravity in this context is power-counting renormalizable as shown by Stelle, but carries massive spin-2 ghost [14]. Furthermore, the quadratic curvature gravity does not resolve the classical singularities, such as cosmological or blackhole singularities. Recently, there has been some interesting progress been made in all these frontiers. One of the issues of resolving the perturbative ghost problem has been addressed in the context of infinite derivative theory of gravity $(I D G)$, see $[15,16]$, and $[17,18]$, which I will review briefly and then discuss how such $I D G$ can also address classical singularities.

\section{Infinite Derivative Gravity $(I D G)$}

The main motivation for an $I D G$ is to address the perturbative ghost, which plagues higher derivative theories in general ${ }^{1}$. The issue of ghost cannot be addressed order by order in higher derivatives. Just to given an example, let us consider a higher derivative massive (non-tachyonic)

\footnotetext{
${ }^{1}$ The issue of ghost is not just pertaining to gravity. The discussion applies to any higher derivative spin-theory, such as spin- 0 , spin-1/2 and spin-1, spin-3/2, etc.
} 
scalar field theory ${ }^{2}$

$$
S=\int d^{4} x \phi \square\left(\square+m^{2}\right) \phi, \Rightarrow \square\left(\square+m^{2}\right) \phi=0, \Rightarrow \Pi\left(p^{2}\right)=\frac{1}{p^{2}\left(p^{2}-m^{2}\right)} \sim \frac{1}{p^{2}}-\frac{1}{p^{2}-m^{2}},
$$

where $\square=\nabla^{\mu} \nabla_{\mu}$ is the d'Alembertian operator, and $\mu=0,1,2,3$. Note that the propagator, $\Pi$, here can be decomposed in such a way that there is a -ve residue, which marks the presence of a massive ghost. One can go for higher orders in derivatives and the problem will persist. Imagine if I had an action, which would yield the equations of motion and the propagator, such that:

$$
S=\int d^{4} x \phi e^{-\frac{\square}{M_{s}^{2}}}\left(\square+m^{2}\right) \phi, \Rightarrow e^{\frac{-\square}{M_{s}^{2}}}\left(\square+m^{2}\right) \phi=0, \Rightarrow \Pi\left(p^{2}\right) \sim \frac{e^{\frac{-p^{2}}{M_{s}^{2}}}}{p^{2}-m^{2}},
$$

then the propagator is suppressed by exponential of an entire function. Since exponential of an entire function does not introduce any pole in the complex plane, besides the original pole mass $m$, there is no question of new dynamical degrees of freedom arising in the spectrum. This is the key observation, which tells us that infinite derivatives do not generate ghost for a massless theory, i.e. $m=0$, case ${ }^{3}$. The results can be verified by using the Hamiltonian approach, following Oströgradsky's prescription. It has recently been demonstrated that Hamiltonian density based on infinite derivatives, i.e. recast as Gaussian kinetic term, can be solely determined by finite number of dynamical degrees of freedom and without any instability [16, 18]. Now based on similar prescription, I will show the results from IDG.

The most general covariant action of higher derivative gravity in 4 dimensions can be recast as [18]:

$$
S=\int d^{4} x \sqrt{-g}\left[\frac{M_{p}^{2}}{2} R+R_{\mu \nu \lambda \sigma} \mathscr{O}_{\alpha \beta \gamma \delta}^{\mu \nu \lambda \sigma} R^{\alpha \beta \sigma \delta}\right],
$$

where $M_{p}=1 / \sqrt{8 \pi G}$ and $\mathscr{O}_{\alpha \beta \gamma \delta}^{\mu \nu \lambda \sigma}$ contains actually infinite covariant operators made up of $\square, \square^{2}, \square^{3}$, $\cdots$, and $R_{\mu \nu \lambda \sigma}$ is the Riemann tensor, which could be replaced by Weyl tensor as well. Note that around Minkowski background the above action gives rise to $\mathscr{O}\left(h^{2}\right)$ action ${ }^{4}$, because each Riemann contributes to $\mathscr{O}(h)$ contribution. Starting from the above action, one can then reduce the above action to ${ }^{5}[18,20]$ :

$$
S=\int d^{4} x \sqrt{-g}\left[\frac{M_{p}^{2}}{2} R+R \mathscr{F}_{1}(\bar{\square}) R+R^{\mu v} \mathscr{F}_{2}(\bar{\square}) R_{\mu \nu}+R^{\mu \nu \lambda \sigma} \mathscr{F}_{3}(\bar{\square}) R_{\mu \nu \lambda \sigma}\right],
$$

with the help of total derivative, Bianchi identities and cyclic properties of Riemann tensor, see for the full prescription of obtaining the action in any constant curvature backgrounds [21, 22]. Note,

\footnotetext{
${ }^{2}$ Metric signature here is mostly plus,,,,-+++ .

${ }^{3}$ Note that according to the Weierstrass theorem any entire function is written as $e^{-\gamma(\square)}$, where $\gamma(\square)$ is an analytic function. For a polynomial $\gamma(\square)$ it is now easy to see that if $\gamma>0$ as $\square \rightarrow \infty$, the propagator is even more convergent than the exponential case leading to non-singular UV behaviour.

${ }^{4} g_{\mu \nu}=\eta_{\mu \nu}+h_{\mu \nu}$, where $\eta_{\mu \nu}$ is the Minkowski metric and $h_{\mu \nu}$ is the perturbation.

${ }^{5}$ In fact one can reduce the above action around a constant curvature backgrounds such as deSitter and anti deSitter backgrounds as well. The only difference will be and addition contribution from cosmological constant $\pm \Lambda$. One can also recast the action in terms of $S_{\mu \nu}=R_{\mu \nu}-(1 / 4) g_{\mu \nu} R$ and the Weyl $C_{\mu \nu \lambda \sigma}$ instead of $R_{\mu \nu \lambda \sigma}$, see [19].
} 
in all these cases the boundary terms have been neglected, if one wishes to take Gibbons-HawkingYork $[23,24]$ boundary action for $I D G$, see [25]. Also note that $\square=\square / M_{s}^{2}$, where $M_{s}$ is the scale of new physics below $M_{p}=2.4 \times 10^{18} \mathrm{GeV}$. In 4 dimensions, $M_{s}$ could be treated as a string scale for instance, or scale of non-locality. The latter will become clear later on.

Some properties of the above $I D G$ action are:

1. 3 form factors: The $\mathscr{F}_{i}$ 's act like form factors, similar to pion form factors in QCD. Is there anyway to constrain them? It has been shown in $[18,19]$ that these form factors are not all independent. In particular, in a Minkowski background one can show that to preserve general covariance, and the massless nature of a graviton would require [18, 20]:

$$
2 \mathscr{F}_{1}(\bar{\square})+\mathscr{F}_{2}(\bar{\square})+2 \mathscr{F}_{3}(\bar{\square})=0 .
$$

Furthermore, for a homogeneous and isotropic background, the Weyl term vanishes, so one can consider $\mathscr{F}_{3}(\bar{\square})=0$ without loss of generality. In fact, one of $\mathscr{F}_{i}(\bar{\square})$ can be set to be zero without loss of any generality. For instance, let us consider $\mathscr{F}_{2}(\square)=0$, then I am left with $\mathscr{F}_{1}(\square)$ and $\mathscr{F}_{3}(\square)$. However, for a cosmological background, which is homogeneous one can even set Weyl/Riemann term to be zero, so the only non-trivial modification arises from $S \sim R+R \mathscr{F}_{1}(\bar{\square}) R$ contribution. In fact, this contribution will be sufficient to show that the Universe can have a nontrivial evolution, a non-singular bouncing cosmology [17]. If I wish to seek a metric potential for a point particle, around Minkowski background, then the Weyl term/Riemann term can be set to zero, therefore we are left with $\mathscr{F}_{1}(\bar{\square})$ and $\mathscr{F}_{2}(\bar{\square})$, such as $S \sim R+R \mathscr{F}_{1}(\bar{\square}) R+R^{\mu v} \mathscr{F}_{2}(\bar{\square}) R_{\mu v}$, this action is precisely used to study non-singular blackhole metric.

2. UV limit: The above action, Eq. (2.4), can be imagined to be a UV complete action. At a classical level it has been shown that the solution of Eq. (2.4) can resolve cosmological singularity for Friedmann-Lemaitre-Roberston-Walker metric [18] ${ }^{6}$. At a quantum level, one can show that a choice for $\mathscr{F}_{1}(\bar{\square})$, and $\mathscr{F}_{2}(\bar{\square})$ are determined by making sure that the propagator for Eq.(2.4) does not have any pole, i.e. the graviton remains massless, general covariance remains intact $[16,17,18]$. I will discuss this issue again in the next subsection.

3. IR limit: The IR limit is taken by $M_{s} \rightarrow \infty$, or $\bar{\square} \rightarrow 0$. In this limit, the above action Eq. (2.4) reduces to pure GR, i.e. the Einstein-Hilbert action. Therefore, in the IR the predictions for the action reduces to that of GR's success story.

\subsection{Graviton Propagator \& Ghost Free Action}

The physical graviton propagator for Eq. (2.4) has been found in Refs. [], which is typically sandwiched between two conserved vertices, i.e. $V^{\mu v} \Pi_{\mu \nu}^{\alpha \beta} V_{\alpha \beta}$, where $V$ 's are the conserved vertices. Here, I summarise the results for the propagator $\Pi$ without the spacetime indices, which can be expressed in terms of the Fourier space, by [18, 20, 26]

$$
\Pi\left(-k^{2}\right)=\frac{P^{(2)}}{a\left(-\bar{k}^{2}\right) k^{2}}+\frac{P^{(0)}}{\left(a\left(-\bar{k}^{2}\right)-3 c\left(-\bar{k}^{2}\right)\right) k^{2}},
$$

\footnotetext{
${ }^{6}$ There is some progress to show that even Kasner type metric can be made singularity free, although the full analytical result is still pending.
} 
where $P^{(2)}$ and $P^{(0)}$ are the spin-projection operators for spin-2 and 0 components, and $\bar{k}=k / M_{s}$, and furthermore $[18,20]$ :

$$
\begin{aligned}
& a\left(-\bar{k}^{2}\right)=1+\frac{1}{2} \mathscr{F}_{2}\left(-\bar{k}^{2}\right)\left(k / M_{p}\right)^{2}+2 \mathscr{F}_{3}\left(-\bar{k}^{2}\right)\left(k / M_{p}\right)^{2}, \\
& c\left(-\bar{k}^{2}\right)=1-2 \mathscr{F}_{1}\left(-\bar{k}^{2}\right)\left(k / M_{p}\right)^{2}-\frac{1}{2} \mathscr{F}_{2}\left(-\bar{k}^{2}\right)\left(k / M_{p}\right)^{2} .
\end{aligned}
$$

Note that there is no vector component, i.e. $P^{(1)}$, is never part of a dynamical degree of freedom in gravity. It decouples from the graviton propagator. Two important observations are:

1. No massive scalar degree of freedom: In order to make sure that the true dynamical degrees of freedom are just massless, transverse and traceless degrees for graviton, I require $a\left(-\bar{k}^{2}\right)=c\left(-\bar{k}^{2}\right)$. This will also ensure that the form of the graviton propagator will look similar to the 4 dimensional GR propagator for Einstein-Hilbert action, but with an interesting modification ${ }^{7}[18,20]$ :

$$
\Pi\left(-k^{2}\right)=\frac{1}{a\left(-\bar{k}^{2}\right)}\left[\frac{P^{(2)}}{k^{2}}-\frac{P^{(0)}}{2 k^{2}}\right],
$$

such that all the information about the infinite derivatives are contained in just one function $a\left(-\bar{k}^{2}\right)$. Note that all the quantities inside the $[\cdots]$ do not introduce any new pole other than the massless, transeverse traceless graviton. The new poles may only come from $a\left(-\bar{k}^{2}\right)$. However, for

$$
a\left(-\bar{k}^{2}\right)=e^{-\gamma\left(k^{2} / M_{s}^{2}\right)},
$$

there will be no poles, and therefore the graviton for Eq. (2.4) remains massless all the way from IR- to- UV. For $M_{s} \rightarrow \infty$, the propagator Eq. (2.9) along with Eq. (2.10) indeed reduces to that of GR, Einstein-Hilbert propagator in 4 dimensions.

This is a strong hint that $I D G$ can indeed provide us an unique way to resolve the ghost problem of Stelle's quadratic curvature gravity. Quantum-loop corrections will not ruin this property due to diffeomorphism invariance ${ }^{8}$. This analysis has been extended to arbitrary $d$ spacetime dimensions [26], and also around dS and AdS backgrounds [21, 22]. Note that the exponentially suppressed

\section{The constraint:}

$$
a\left(-\bar{k}^{2}\right)=c\left(-\bar{k}^{2}\right)
$$

suggests that the three form factors, i.e. $\mathscr{F}_{i}$ 's, are not independent: ${ }^{9}$ see Eq. (2.5). However, this provides us some freedom, if I select $\mathscr{F}_{3}(\bar{\square})=0$, then: $2 \mathscr{F}_{1}(\bar{\square})+\mathscr{F}_{2}(\bar{\square})=0$. A very similar constraint would be derived if in the original action Eq. (2.4) were written in terms of Weyl instead of Riemann. See footnote 8.

\footnotetext{
${ }^{7}$ The GR propagator has the following form in 4 dimensions: $\Pi\left(-k^{2}\right)=\frac{P^{(2)}}{k^{2}}-\frac{P^{(0)}}{2 k^{2}}$. [13].

${ }^{8}$ This point has been stressed repeatedly by Tomboulis [16, 27], and also by Biswas [28].

${ }^{9}$ If I were working with Weyl term instead of Riemann, the constraint $a\left(-\bar{k}^{2}\right)=c\left(-\bar{k}^{2}\right)$ would lead to following constraint: $6 \mathscr{F}_{1}(\bar{\square})+3 \mathscr{F}_{2}(\bar{\square})+2 \mathscr{F}_{3}(\bar{\square})=0$, see [19].
} 
Now, along with Eqs. $(2.7,2.10)$ and the above constraint, the $I D G$ action can be written as $[18,19]$ :

$$
S=\int d^{4} x \sqrt{-g}\left[\frac{M_{p}^{2}}{2} R+M_{p}^{2} R\left(\frac{e^{-\gamma(\bar{\square})}-1}{\square}\right) R-2 M_{p}^{2} R^{\mu \nu}\left(\frac{e^{-\gamma(\bar{\square})}-1}{\square}\right) R_{\mu \nu}\right]
$$

Now, one can select a specific form of $\gamma(\bar{\square})$. The simplest choice will be

$$
\gamma(\bar{\square})=\bar{\square}=\square / M_{s}^{2},
$$

In fact, one can select a wider range of entire function. The most general case can be expressed in terms of polynomials, and these issues have been discussed in Refs. [29].

Now, with the above construction of $I D G$ action, Eq. (2.12), I can explore various cosmological, astrophysical and field theory applications. The complete equations of motion for Eq. (2.4) have been obtained in [19], and indeed Eq. (2.12) will be a particular case.

\section{Classical Singularities}

Let us first explore some classical singularities, and demonstrate how IDG can ameliorate classical singularity problems persisting in GR. In GR a point like source generates a metric which leads to a singular Schwarzschild metric. At $r \rightarrow 0$ the metric potential blows up to infinity, and also the Kretschmann scalar. One of the outcome of this metric is the presence of an event horizon at $r=2 G M$, where $G$ is the Newton's constant, and $M$ is the mass of a point source. At the event horizon the light-cone gets stretched. The metric potential, $\Phi=G M / r$, becomes large for $r<G M$, and for $r>G M$, the metric potential is always less than unity. An IR observer always sees only the perturbed Newtonian potential $\Phi \leq 1$, until the observer reaches very close to the event horizon. At $r=0$, there is indeed a curvature singularity, the Kretschmann scalar blows up at $r \rightarrow 0$. Could $I D G$ with an action Eq. (2.12) resolve such a singularity? - the answer is yes! ${ }^{10}$

\subsection{Non-singular blackhole solution}

Let us consider a simple static metric where the metric potentials: $2 \Phi, 2 \Psi \ll 1$

$$
d s^{2}=-(1-2 \Phi) d t^{2}+(1+2 \Psi) d r^{2}
$$

for a point source, say $M$. The potentials $\Phi, \Psi$ can be solved analytically for Eq. (2.12) [18] ${ }^{11}$

$$
\Phi(r)=\Psi(r)=-\frac{M}{M_{p}^{2} r} \sqrt{\frac{\pi}{2}} \operatorname{erf}\left(\frac{M_{s} r}{2}\right)
$$

\footnotetext{
${ }^{10}$ In this regard, ghost free IDG action Eq. (2.12) reminds us of Born-Infeld [30] extension of Maxwell's electromagnetism. In Maxwell's theory also there exists a singular Coulomb potential for a point-charge particle. The self energy of an electron for instance blows up. However, Born-Infeld action is a non-linear extension of Maxwell's linear theory, where there is a parameter very similar to $M_{S}$, below which the theory behaves as that of Maxwell's. In Born-Infeld theory the self energy of an electron is finite and it does not blow up. A similar challenge arises for Einstein-Hilbert's action, the question is - could we then find an analogue of Born-Infeld gravity? IDG provides an answer to this fundamental quantion, as I will demonstrate below.

${ }^{11} I D G$ kind of action will avoid blackhole singularity has also been discussed before in the context of $\alpha^{\prime}$ corrections in string theory $[31,32]$.
} 
This potential is finite near $r \rightarrow 0$, and decays as $1 / r$ at distances $r \gg M_{s}^{-1}$. The table-top tests of $1 / \mathrm{r}$ fall of Newtonian gravity has been tested in the laboratory up to $5.6 \times 10^{-5} \mathrm{~m} \mathrm{[33]}$, which implies that $M_{s}>0.004 \mathrm{eV}$. One can also test other examples of $\gamma(\bar{\square})$, and place similar constraints on $M_{s}$ [29]. One can further compute Kretschmann scalar for the above metric potentials and it is indeed finite.

By observing Eq. (3.2), and for $r<M_{s}^{-1}$, the potential becomes constant, because erf grows linearly in argument, i.e. $\operatorname{erf}\left(M_{s} r / 2\right) \sim r M_{s} / 2$, therefore it cancels $1 / r$ factor in the potential. A constant Newtonian potential would lead to a vanishing gravitational force: $F_{g} \rightarrow 0$. Classically the action Eq. (2.12) becomes asymptotically free, for $\gamma(\square)=\square / M_{s}^{2}$, similar in spirit as QCD. Where above the energies of de-confining phase both quarks and gluons become free! Indeed, one has to show what happens at the quantum level.

So far, I have discussed a static scenario for a metric potential. However, in reality the dynamics of a collapse of matter becomes an important issue. Frolov and Zelnikov [34, 35, 36, 37] have studied the dynamical problem of colliding two ultra-relativistic wavefronts to see whether apparent horizon forms for the IDG action Eq. (2.12) with Eq. (2.13). What the authors have found is very interesting - the ultra-relativistic collision does not lead to formation of a singularity or the event horizon. The latter can be understood easily, note that both the metric potentials in Eq. (3.1) are bounded by unity, $2 \Phi=2 \Psi \leq 1$, so effectively there is no event horizon for such a system.

Since, $M_{s}$ is very large, if it is typically of the order of GUT scale or close to Planck scale, it is interesting to seek the validity of the linear approximation, i.e. the metric potentials are bounded by unity? There are couple of lessons to be learnt here.

1. Validity of the non-singular solution: Indeed mass of a blackhole $M_{b h}$, cannot be arbitrary large for $2 \Phi=2 \Psi \leq 1$. In fact, the assumption is valid only up to [18]:

$$
M_{b h} \leq \mathscr{O}(1) \frac{M_{p}^{2}}{M_{s}} .
$$

Indeed this places a limit on $M_{b h}$, if $M_{s} \sim M_{p}$ then the allowed mass range for $M_{b h} \leq M_{p} \sim$ $10^{-5}$ grams. For such a small point source, indeed this theory has no singularity. The constraints from a current table top limit on $M_{s} \geq 10^{-2} \mathrm{eV}$, then yields a non-singular blackhole (or non-singular compact object (NSCO) ) mass constraint around $M_{b h} \leq 10^{25}$ grams. This is an interesting number, because for this range of blackhole/NSCO mass can be probed by many astrophysical means, and such objects, at least in the context of GR can be produced in the early Universe via phase transition [38]. In GR indeed such massive blackholes are stable under Hawking radiation, and can play a significant role in structure formation and history of the Universe as a dark matter candidate.

In $I D G$ for Eqs. $(2.12,2.13,3.1)$, such massive objects, i.e. $M \leq 10^{25}$ grams with $M_{s} \geq$ $10^{-2} \mathrm{eV}$ will never form a singular system with event horizon. Such NSCO may gravitationally bound - very weakly, and may not act as a dark matter candidate. The reason is that these NSCO can be probed by high energy photons, and lack of singularity means that very ultra high energy photons can indeed probe inner structure of the system.

Indeed, for such NSCO there will be no information loss paradox ever, since there is no formation of event horizon. One can still discuss gravitational entropy of the system, and it 
has been shown to be following the Area-Law with minor correction. This computation has been performed in Ref. [39].

2. Fate of supermassive astrophysical blackholes: In Einstein's GR one can expect supermassive blackholes residing in the centre of our galaxy, roughly with mass $\geq 10^{33}$ grams. This number is more than 8-orders of magnitude bigger than one would expect from NSCO from $I D G$ with $M_{s} \geq 10^{-2} \mathrm{eV}$. The obvious question is - do astrophysical blackholes have singularity and the event horizon within $I D G$ ?

Of course, at this moment there is no full nonlinear solution for the metric Eq. (3.1) for Eq. $(2.12,2.13)$. Nevertheless, one can still conclude very interesting consequences. If in the process of collapse, the metric potential remains weak, i.e. $2 \Phi, 2 \Psi \leq 1$, the linear approximations will always be valid. Furthermore, if the gravitational interaction becomes weak at short distances, it can avoid forming a singularity, or avoid forming a trapped surface by defocusing the null or time-like rays. This will also mean that the formation of apparent horizon can be avoided, at least in the context of non-rotating scenario. Note that in a static and spherically symmetric metric, the apparent horizon and the event horizon match. If the event horizon can be avoided this means that irrespective of the mass of a supermassive blackhole, the two metric potentials, $2 \Phi, 2 \psi \leq 1$, will always be less than 1 through out the inside of the radius $r_{g}=2 G M$. There are some smoking gun hints which suggest that gravity becomes weak in the UV in $I D G$ and never allow the metric potentials to exceed beyond 1. I will discuss this case very briefly.

\subsection{Non-singular cosmological solution}

In Einstein's GR, cosmological Big Bang singularity is inevitable as $t \rightarrow 0^{12}$, where the energy density of the Universe blows up. There have been many attempts to resolve cosmological singularity within GR, plus matter source, but all these attempts inevitably violate either one or all of the matter energy conditions, i.e. strong, weak, or null energy conditions ${ }^{13}$. The aim is to obtain a non-singular cosmology without violating any of the energy conditions in the matter sector or in the gravitational sector. In the latter case, for instance action, $S \sim M_{p}^{2} R-R^{2}$ will also resolve the singularity problem, but at the cost of a ghost, i.e. -ve kintic term for $R^{2}$.

The $I D G$ provides a very intriguing solution, gravity at small time scales becomes so weak that Universe could bounce back - resolving the cosmological singularity problem in an elegant fashion. In fact, the above action Eq. (2.12) with Eq. (2.13) gave rise to a non-singular bouncing

\footnotetext{
${ }^{12}$ Here I am mostly talking about a flat spatial background. This is the most challenging case along with +ve spatial curvature. The -ve spatial curvature is rather easy to resolve the singularity problem even within GR. It is believed that inflation might delay the cosmological singularity, and it is true, it pushes back the singularity in time, but it fails to address cosmological singularity [40].

${ }^{13}$ Loop quantum cosmology attempts to address the singularity problem, but in a mini-superspace where the bouncing solution is constructed without studying the entire phase space [10]. In principle, one should study the problem beyond mini-superspace approximation and show whether singularity can be resolved or not. While string theory does not directly shed light on extremely high curvature regimes in time dependent background, also one would expect string field theory to play a certain role, in particular closed string field theory. In this regard IDG captures certain aspects of string field theory, higher derivatives are akin to $\alpha^{\prime}$ corrections [31, 32].
} 
cosmology in a linear regime with an oscillating solution around Minkowski background [18]:

$$
d s^{2}=-d t^{2}+(1+A \sin (\lambda t))\left(d x^{2}+d y^{2}+d z^{2}\right), \quad A \ll 1,
$$

where $A$ is constant amplitude of the oscillations. This is an analogue of a gravitational soliton. Such a solution is not possible within GR. Even to obtain this solution, it was realised that one requires an additional condition on gravitational degrees of freedom [18].

1. An additional ghost free spin-0 degree of freedom: Besides massless graviton, an additional degree of freedom is required. In order to resolve the cosmological singularity, one requires $[18,41]$

$$
a\left(-\bar{k}^{2}\right) \neq c\left(\bar{k}^{2}\right),
$$

which means that there is an additional pole in the spin- 0 component. In fact this pole is harmless, what it means is that there is an additional scalar degree of freedom which is required to resolve the cosmological singularity. In fact, any $f(R)$ theory does have a BransDicke scalar mode [42]. But, not any kind of $f(R)$ gravity would give rise to a non-singular cosmology.

For instance, action: $S \sim M_{p}^{2} R+R^{2}$ theory does not give rise to a non-singular bounce, but $S \sim M_{p}^{2} R-R^{2}$ theory does give rise to a bouncing cosmology [43], but at the cost of a ghost degree of freedom. Therefore the right question to ask - what should be the form of $c(\bar{\square})$ or $c\left(-\bar{k}^{2}\right)$, such that there is just an additional pole, and it is ghost free?

In order to accommodate one extra pole in the spin- 0 component of the propagator, an appropriate condition will be then $[18,41]$ :

$$
c(\bar{\square})=\frac{a(\bar{\square})}{3}\left[1+2\left(1-\frac{\square}{m^{2}}\right) \widetilde{a}(\bar{\square})\right],
$$

and the corresponding propagator becomes [18, 41] (also see the thesis [44]):

$$
\Pi\left(-k^{2}\right)=\frac{1}{a\left(-\bar{k}^{2}\right)}\left[\frac{P^{(2)}}{k^{2}}-\frac{1}{\widetilde{a}\left(-\bar{k}^{2}\right)}\left(\frac{p^{(0)}}{k^{2}}-\frac{p^{(0)}}{k^{2}+m^{2}}\right)\right] .
$$

It is evident that there is an additional scalar pole with mass $m$. This will serve as an effective mass for the Brans-Dicke scalar. Now, what should be the corresponding IDG action which would give rise to a new propagator Eq. (3.7)? I will discuss this below.

2. The $I D G$ action which resolves cosmological singularity: We have already discussed that the 3 form factors of the original IDG action, Eq. (2.4), are not all independent, see Eq. (2.5). There is a bit of a freedom which one can exploit. For instance in a homogeneous and isotropic background, it is sufficient to study an $I D G$ action which contains $S \sim$ $M_{p}^{2} R+R \mathscr{F}_{1}(\bar{\square}) R$. The reason is very simple, I can set $\mathscr{F}_{2}=0$, leaving behind $\mathscr{F}_{1}, \mathscr{F}_{3} \neq 0$. However, for a homogeneous and isotropic background the Weyl term can be set zero at the background level, so the IDG action can be recast completely by [17]:

$$
S=\int d^{4} x \sqrt{-g}\left[\frac{M_{p}^{2}}{2} R+M_{p}^{2} R\left(\frac{e^{-\square / M_{s}^{2}}-1}{\square}\right) R\right] .
$$


The equations of motion for this action has been studied in [17, 45]. The equations of motion satisfy an interesting recursive solution for 2 constants, $c_{1}, c_{2}$ :

$$
\square R=c_{1} R+c_{2},
$$

which solves the full non-linear equations of motion of the above action Eq. (3.8), and yields a very interesting solution in presence of a +ve cosmological constant: $\Lambda$ [17, 45]:

$$
d s^{2}=-d t^{2}+a_{0} \cosh ^{2}(\lambda t)\left[d x^{2}+d y^{2}+d z^{2}\right], \quad \lambda=\sqrt{\frac{\Lambda}{3 M_{p}^{2}}}, \quad \Lambda \sim M_{s}^{4} .
$$

The scale factor is given by $\cosh (\lambda t)$, which has both positive and negative branches, means that the solution is time symmetric. The solution indeed replaces the singularity present in homogeneous and isotropic Universe in GR by a non-singular bouncing Universe. The bounce solution is robust under perturbations, for both sub-Hubble and super-Hubble perturbations, see $[46,47]$.

3. Defocusing of null rays and Raychoudhuri equation: Avoidance of cosmological singularity has now been tested by studying null/time-like geodesics, and how they get defocused for the above action Eq. (3.8) for normal matter, which does not violate any of the energy conditions, i.e. strong, weak, null energy conditions [41, 48]. This is due to the fact that IDG modifies the Raychoudhuri's equation, compared to that in the GR case, in such a way that the expansion parameter $\theta=\nabla_{\mu} k^{\mu}$, where $k^{\mu}$ is the null ray, follows:

$$
\frac{d \theta}{d \tau}+\frac{1}{2} \theta^{2} \geq 0
$$

where $\tau$ is the affine parameter. The expansion parameter defocuses in presence of $I D G$, which shows the matter never gets trapped in a finite region of spacetime, and the manifold remains smooth. As such the defocusing theorem of null rays does not shed light on spacetime singularity, but it ensures that the space-time manifold is regular and null geodesics are past-complete.

Furthermore, the above action Eq. (3.8) is a UV complete action for a quadratic curvature inflation, as pointed out in Refs. [49]. This action has very intriguing B-mode prediction, which can produce a range of tensor-to-scalar ratio $0.001 \leq r \leq 0.6[50]$.

\section{Quantum UV finiteness}

$I D G$ provides an intriguing quantum feature; being an infinite derivative theory of gravity, and with an exponentially suppressed propagator Eq. $(2.9,2.10)$, at higher loops one would expect gravity should be finite. In fact, gravity should become asymptotically free in the UV. Since the propagator is exponentially suppressed, in the context of gravity, which has only derivative interactions, the vertex operator for 3 incoming momenta will be schematically given by [51]:

$$
V\left(\bar{k}_{i}\right) \sim C\left(\bar{k}_{i}^{2}\right) f\left(e^{\bar{k}_{i}^{2}}\right)
$$


where $i=1,2,3$ and $C\left(\bar{k}_{i}^{2}\right)$ is a polynomial function of $k_{1}, k_{2}, k_{3}$, and $f\left(\bar{k}_{i}^{2}\right)$ is an exponential function of some linear combination of $\bar{k}_{i}^{2}$. In the UV limit, certainly $f\left(\bar{k}_{i}^{2}\right)$ will dominates over the polynomial, therefore, the counting of superficial degree of divergence will depend solely on exponentially suppressed propagator and exponentially enhanced vertices in the UV. Couple of crucial points to note here:

1. Non-local interactions: An important point to note here is that the vertex operator is spread over a region of space time, which yields non-local interactions, i.e. $e^{\bar{k}^{2}}$, essentially in the UV $k \gg M_{s}$, or within space-time $M_{s}^{-1}$. While in the IR, for $k \ll M$ or space-time region $\gg M_{s}^{-1}$ the interactions become local. In the decoupling limit when $M_{s} \rightarrow \infty$, the gravitational action reduces to that of a pure GR action, see Eq. (2.12). A further point to note here is that the free theory is always local, and non-locality arises only in interactions.

2. Renormalizability: A detailed power counting argument shows that the degree of divergence is given by ${ }^{14}[15,51,52]$ :

$$
D=1-L,
$$

where $L$ stands for loops. For $L=1$, the action Eq. (2.12) gives rise to divergence, which is similar to that of GR at $L=1$. One requires counter term to absorb the divergence. However, for $L>1$, the superficial degree of divergence is negative, suggests that the theory in UV should be finite, and power counting renormalizable. This means that higher loops, $L>1$, gives rise to a finite result without any divergences with respect to internal-loop momentum and external-loop momenta. If this is the case, then the theory becomes asymptotically free, where gravitational interactions become weaker in the UV. A glimpse of this has already been seen at a classical level for Eq. (2.13), the metric potential Eq. (3.2) gave rise to a constant potential for $r<M_{s}^{-1}$, and net force between two masses vanish, i.e. $F_{g} \rightarrow 0$.

Indeed, this conclusion is extremely interesting and bears some resemblance to Weinberg's idea of asymptotic safety []. nevertheless, its worth spending more time in understanding the subtle issues and show rigorously by doing some explicit computations.

In this review, I will not discuss any further the gravitational interaction, instead just to build an intuition - I will briefly discuss the case of a scalar toy model which will resemble gravity to a large extent with non-local interactions. Inspired by this infinite-derivative gravitational action, see Eq. (2.4), we have formulated a scalar toy model in Ref. [51] that captures the essential features of the UV behaviour of the IDG action, Eq. (2.4). The scalar toy model action is given by

$$
S_{\text {scalar }}=S_{\text {free }}+S_{\text {int }},
$$

where ${ }^{15}$

$$
S_{\text {free }}=\frac{1}{2} \int d^{4} x(\phi \square a(\square) \phi)
$$

\footnotetext{
${ }^{14}$ From the power counting argument, the superficial degree of divergence in GR is: $D=2 L+2$, so at higher loops $L>1$, the superficial degree of divergence keeps increasing. At one-loop, 2 derivative GR has divergences, but it can be absorbed by local counter-terms. At a quadratic order in gravity, the action considered by Stelle in [14] is indeed power counting renormalizable.

${ }^{15}$ One way to obtain the scalar toy model is to exploit the symmetry of GR, a global scaling symmetry, $g_{\mu \nu} \rightarrow \lambda g_{\mu \nu}$, and around the Minkowski background, $g_{\mu v}=\eta_{\mu v}+h_{\mu \nu}$, the scaling symmetry translates to $h_{\mu v} \rightarrow(1+\varepsilon) h_{\mu \nu}+\varepsilon \eta_{\mu \nu}$.
} 
and

$$
S_{\mathrm{int}}=\frac{1}{M_{P}} \int d^{4} x\left(\frac{1}{4} \phi \partial_{\mu} \phi \partial^{\mu} \phi+\frac{1}{4} \phi \square \phi a(\bar{\square}) \phi-\frac{1}{4} \phi \partial_{\mu} \phi a(\bar{\square}) \partial^{\mu} \phi\right) ;
$$

we have $a(\bar{\square})=e^{-\bar{\square}} \equiv e^{-\square / M^{2}}$. The equation of motion for the action given by Eq. 4.3 satisfies the shift-scaling symmetry $\phi \rightarrow(1+\varepsilon) \phi+\varepsilon$, where $\varepsilon$ is infinitesimal.

Many interesting properties of Eq. (4.3) can be established, which I summarise below:

1. Global symmetry as opposed to local symmetry: The above symmetry $\phi \rightarrow(1+\varepsilon) \phi+\varepsilon$ is a global symmetry as opposed to a diffeomorphism symmetry, which is local. As a result, the scalar-toy model will have issues such as generating effective mass at one-loop, which will never occur in the case of gravity, diffeomorphism invariance will protect the massless ness of a graviton. See for details [51].

2. One-loop divergence and local counter term: There exists a divergence with respect to internal- loop momentum form 1-loop 2-point function. This can be cured by adding a counter term which is local, containing no infinite derivative terms, i.e. $a(\bar{\square})$. In this respect Eq. (4.3) can be made one loop renormalizable.

3. Higher loops and UV finite: In Ref. [51] it was established that the dressed propagator is more exponentially suppressed than the bare propagator, and the dressed vertices behave as exponentials of external momenta when the external momenta are large. By employing dressed propagators and vertices, $n$-loop, 2- \& 3-point diagrams constructed out of lowerloop, 2- \& 3-point diagrams become finite in the UV with respect to internal loop momentum, and no UV divergences arise and no new counterterm is required.

Similarly, by employing dressed vertices and propagators, $n$-loop, $N$-point diagrams constructed out of lower-loop 2- \& 3-point diagrams are also UV finite with respect to internal loop momentum. Also, the external momentum dependences of $n$-loop, $N$-point diagrams constructed out of lower-loop 2- \& 3-point diagrams decrease as the loop-order increases and the external momentum divergences are eliminated at sufficiently high loop-order [51].

4. Scattering amplitude and astrophysical blackhole: In Ref. [53] the UV behaviour of scattering diagrams were investigated for Eq. (4.3), and it was established that the external momentum dependence of the scattering diagrams is convergent for large external momenta. The results were obtained by dressing the bare vertices of the scattering diagrams by considering renormalised propagator and vertex loop corrections to the bare vertices. With an increasing loop order, the exponents in the dressed vertices become negative at sufficiently high loop-order, and the corresponding amplitude decreases exponentially. All these results show that trans-Planckian scattering is not a problem for infinite derivative theories. The decrease in scattering amplitude suggests that these theories behave better in the UV, similar conclusions were also obtained in Ref. [28], where it was assumed that the scalar field $\phi$ could take the role of a Standard Model Higgs, and also in thermal field theory [54], which has been helpful to understand Hagedorn phase transition.

For multi-particle scatterings, the amplitude scales as $\sim e^{-\sqrt{n} p^{2} / M_{s}^{2}}$, where $n$ is the number of external legs, assuming that all the momenta are the same, i.e. $p$. This may have a 
profound conseqence for astrophysical blackholes. What it means is that any astrophysical blackhole which is typically constructed out of $n$ quanta, in this particular case $n$-bosonic states. However, as we bring these states closer to each other - they are bound to interact and scatter, but their scattering amplitude decreases exponentially with the number of states. This will then give rise to an effective scale of non-locality to be:

$$
M_{e f f} \sim \frac{M_{s}}{\sqrt{n}}
$$

larger than the original $M_{s}$. For instance, although the scale of non-locality is $M_{s}^{-1}$, but its effect can be enlarged in presence of multiple scattering states by a larger region of spacetime, i.e. $\sqrt{n} M_{s}^{-1}$. This argument might prevent a complete collapse of matter under gravity, and prevent forming a blackhole alltogether. In reality, one can imagine now $\phi$ being replaced by $h_{\mu \nu}$, and imagining that the end stage of a blackhole is constituted by graviton.

\section{5. conclusion}

I have summarised here some interseting results emerging from infinite derivative field theories and gravity. In particular, I have discussed how to constrain an IDG action from diffeomorphism invariance, causality, and stability arguments, such as ghost free consideration. I have explored important properties of $I D G$ at a classical level, in particular resolving singularity in mini-blackholes, and cosmological Big Bang singularity problems. At a quantum level, I have discussed power counting argument to show that a class of theory in gravity can be made UV finite, or asymptotically free. I have discussed a scalar-toy model which captures essence of IDG, and in this context I have argued how such infinite derivative scalar field theory can be made UV finite [51].

There are also very important challenges ahead - such as the fate of astrophysical blackholes, existence of singularity, and presence/absence of event horizon. At a quantum front, indeed one would like to show that Eq. (2.12) is indeed UV finite, and a viable theory of quantum gravity.

\section{References}

[1] C. M. Will, Living Rev. Rel. 17, 4 (2014) [arXiv:1403.7377 [gr-qc]].

[2] B. P. Abbott et al. [LIGO Scientific and Virgo Collaborations], Phys. Rev. Lett. 116, no. 6, 061102 (2016) [arXiv:1602.03837 [gr-qc]].

[3] G. 't Hooft and M. J. G. Veltman, Ann. Inst. H. Poincare Phys. Theor. A 20, 69 (1974).

[4] S. W. Hawking and G. F. R. Ellis,

[5] B. S. DeWitt, Phys. Rev. 160 (1967) 1113.

[6] B. S. DeWitt, Phys. Rev. 162, 1195 (1967).

[7] B. S. DeWitt, Phys. Rev. 162 (1967) 1239.

[8] S. Weinberg. Ultraviolet Divergences In Quantum Theories Of Gravitation - Hawking, S.W. (Cambridge Univ. (UK)); Israel, W. (Alberta Univ., Edmonton (Canada). Theoretical Physics Inst.) (eds.); p. 790-831; ISBN 0521 22285 0; 1979; p. 790-831; University Press; Cambridge. 
[9] J. Ambjorn, A. Goerlich, J. Jurkiewicz and R. Loll, Phys. Rept. 519, 127 (2012) [arXiv:1203.3591 [hep-th]].

[10] A. Ashtekar, Lect. Notes Phys. 863 (2013) 31 [arXiv:1201.4598 [gr-qc]].

[11] J. Polchinski. String theory. Vol. 2: Superstring theory and beyond - 1998. Univ. Pr.. Cambridge, UK: r. 531p.

[12] M. Ostrogradski. Memoires sur les equations differentielles relatives au probleme des isoperimetres, Mem. Ac. St. Petersbourg VI (1850) 385.

[13] P. Van Nieuwenhuizen, Nucl. Phys. B 60, 478 (1973).

[14] K. S. Stelle, Phys. Rev. D 16, 953 (1977).

[15] E. Tomboulis, Phys. Lett. 97B, 77 (1980).

[16] E. T. Tomboulis, hep-th/9702146.

[17] T. Biswas, A. Mazumdar and W. Siegel, JCAP 0603, 009 (2006) [hep-th/0508194].

[18] T. Biswas, E. Gerwick, T. Koivisto and A. Mazumdar, Phys. Rev. Lett. 108, 031101 (2012) [arXiv:1110.5249 [gr-qc]].

[19] T. Biswas, A. Conroy, A. S. Koshelev and A. Mazumdar, Class. Quant. Grav. 31 (2014) 015022 Erratum: [Class. Quant. Grav. 31 (2014) 159501] [arXiv:1308.2319 [hep-th]].

[20] T. Biswas, T. Koivisto and A. Mazumdar, "Nonlocal theories of gravity: the flat space propagator," arXiv:1302.0532 [gr-qc].

[21] T. Biswas, A. S. Koshelev and A. Mazumdar, Fundam. Theor. Phys. 183, 97 (2016) [arXiv:1602.08475 [hep-th]].

[22] T. Biswas, A. S. Koshelev and A. Mazumdar, Phys. Rev. D 95, no. 4, 043533 (2017) [arXiv:1606.01250 [gr-qc]].

[23] J. W. York, Jr., Phys. Rev. Lett. 28, 1082 (1972).

[24] G. W. Gibbons and S. W. Hawking, Phys. Rev. D 15 (1977) 2752.

[25] A. Teimouri, S. Talaganis, J. Edholm and A. Mazumdar, JHEP 1608, 144 (2016) [arXiv:1606.01911 [gr-qc]].

[26] A. Conroy, A. Mazumdar, S. Talaganis and A. Teimouri, Phys. Rev. D 92, no. 12, 124051 (2015) [arXiv:1509.01247 [hep-th]].

[27] E. T. Tomboulis, Phys. Rev. D 92, no. 12, 125037 (2015) [arXiv:1507.00981 [hep-th]].

[28] T. Biswas and N. Okada, Nucl. Phys. B 898, 113 (2015) [arXiv:1407.3331 [hep-ph]].

[29] J. Edholm, A. S. Koshelev and A. Mazumdar, Phys. Rev. D 94, no. 10, 104033 (2016) [arXiv:1604.01989 [gr-qc]].

[30] M. Born and L. Infeld, Proc. Roy. Soc. Lond. A 144, 425 (1934).

[31] A. A. Tseytlin, Phys. Lett. B 363, 223 (1995) [hep-th/9509050].

[32] W. Siegel, hep-th/0309093.

[33] D. J. Kapner, T. S. Cook, E. G. Adelberger, J. H. Gundlach, B. R. Heckel, C. D. Hoyle and H. E. Swanson, Phys. Rev. Lett. 98 (2007) 021101 [hep-ph/0611184]. 
[34] V. P. Frolov and A. Zelnikov, Phys. Rev. D 93, no. 6, 064048 (2016) [arXiv:1509.03336 [hep-th]].

[35] V. P. Frolov, Phys. Rev. D 94, no. 10, 104056 (2016) [arXiv:1609.01758 [gr-qc]].

[36] V. P. Frolov, Phys. Rev. Lett. 115, no. 5, 051102 (2015) [arXiv:1505.00492 [hep-th]].

[37] V. P. Frolov, A. Zelnikov and T. de Paula Netto, JHEP 1506, 107 (2015) [arXiv:1504.00412 [hep-th]].

[38] B. J. Carr, eConf C 041213, 0204 (2004) [astro-ph/0504034].

[39] A. Conroy, A. Mazumdar and A. Teimouri, Phys. Rev. Lett. 114, no. 20, 201101 (2015) [arXiv:1503.05568 [hep-th]].

[40] A. Mazumdar and J. Rocher, Phys. Rept. 497, 85 (2011) [arXiv:1001.0993 [hep-ph]].

[41] A. Conroy, A. S. Koshelev and A. Mazumdar, JCAP 1701, no. 01, 017 (2017) [arXiv:1605.02080 [gr-qc]].

[42] C. Brans and R. H. Dicke, Phys. Rev. 124, 925 (1961).

[43] A. A. Starobinsky, Phys. Lett. 91B, 99 (1980).

[44] A. Conroy, "Infinite Derivative Gravity: A Ghost and Singularity-free Theory," arXiv:1704.07211 [gr-qc].

[45] A. S. Koshelev and S. Y. Vernov, Phys. Part. Nucl. Lett. 11, no. 7, 960 (2014) [arXiv:1406.5887 [gr-qc]].

[46] T. Biswas, T. Koivisto and A. Mazumdar, JCAP 1011, 008 (2010) [arXiv:1005.0590 [hep-th]].

[47] T. Biswas, A. S. Koshelev, A. Mazumdar and S. Y. Vernov, JCAP 1208, 024 (2012) [arXiv:1206.6374 [astro-ph.CO]].

[48] A. Conroy, A. S. Koshelev and A. Mazumdar, Phys. Rev. D 90, no. 12, 123525 (2014) [arXiv:1408.6205 [gr-qc]].

[49] T. Biswas and A. Mazumdar, Class. Quant. Grav. 31, 025019 (2014) [arXiv:1304.3648 [hep-th]]. D. Chialva and A. Mazumdar, Mod. Phys. Lett. A 30, no. 03n04, 1540008 (2015) [arXiv:1405.0513 [hep-th]]. B. Craps, T. De Jonckheere and A. S. Koshelev, JCAP 1411, no. 11, 022 (2014) [arXiv:1407.4982 [hep-th]]. A. S. Koshelev, K. Sravan Kumar and P. Vargas Moniz, arXiv:1604.01440 [hep-th].

[50] J. Edholm, Phys. Rev. D 95, no. 4, 044004 (2017) [arXiv:1611.05062 [gr-qc]].

[51] S. Talaganis, T. Biswas and A. Mazumdar, Class. Quant. Grav. 32, no. 21, 215017 (2015) [arXiv:1412.3467 [hep-th]]. S. Talaganis and A. Mazumdar, “Towards UV Finiteness of Infinite Derivative Theories of Gravity and Field Theories," arXiv:1704.08674 [hep-th].

[52] L. Modesto, Phys. Rev. D 86, 044005 (2012) [arXiv:1107.2403 [hep-th]].

[53] S. Talaganis and A. Mazumdar, Class. Quant. Grav. 33, no. 14, 145005 (2016) [arXiv:1603.03440 [hep-th]].

[54] T. Biswas, J. A. R. Cembranos and J. I. Kapusta, Phys. Rev. Lett. 104, 021601 (2010) doi:10.1103/PhysRevLett.104.021601 [arXiv:0910.2274 [hep-th]]. T. Biswas, J. A. R. Cembranos and J. I. Kapusta, JHEP 1010, 048 (2010) [arXiv:1005.0430 [hep-th]]. T. Biswas, J. A. R. Cembranos and J. I. Kapusta, Phys. Rev. D 82, 085028 (2010) [arXiv:1006.4098 [hep-th]]. T. Biswas, J. Kapusta and A. Reddy, JHEP 1212, 008 (2012) [arXiv:1201.1580 [hep-th]]. 